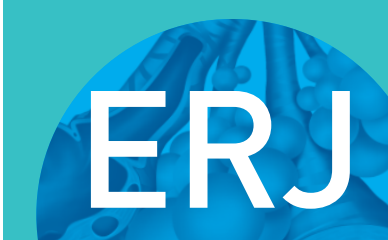

open research

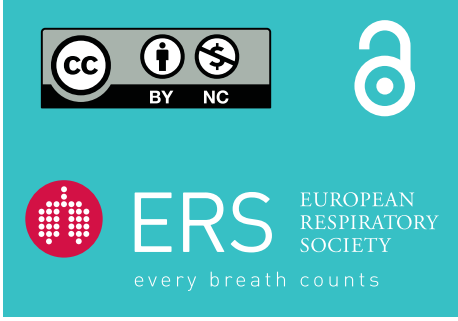

\section{Proteomic profiling of peripheral blood and bronchoalveolar lavage fluid in interstitial lung diseases: an explorative study}

\section{To the Editor:}

Interstitial lung diseases (ILDs) are a diverse group of disorders, which differ significantly with respect to aetiopathogenesis, clinical, radiological, and pathological picture. Idiopathic pulmonary fibrosis (IPF) and sarcoidosis represent two distinctive examples of ILDs [1].

In IPF, the most significant pathobiological mechanism is linked to the uncontrolled activity of various growth factors resulting in accumulation of myofibroblasts and excessive secretion of extracellular matrix (ECM) proteins, mainly collagen. As a consequence, we observe irreversible and progressive fibrotic lung disease, leading to death within $3-5$ years in $50 \%$ of patients [2].

By contrast, sarcoidosis has an immunoinflammatory systemic pathology, with T-helper (Th)1 lymphocyte predominance and excess production of interferon- $\gamma$, leading to granuloma formation in various organs. Löfgren syndrome is a clinical phenotype of acute sarcoidosis, with a very low risk of lung fibrosis, a high rate of spontaneous resolution, and a good overall prognosis [3].

No studies on simultaneous proteome analysis of serum and bronchoalveolar lavage fluid (BALF) in IPF and sarcoidosis have been published to date. It is noteworthy that there are few comparative studies of different ILDs using BALF [4]. Proteomics using panels targeted at various biological processes and other techniques that are more affordable than mass spectrometry, such as proximity extension assay (PEA), are becoming more readily available [5]. Briefly, 92 oligonucleotide labelled antibody probe pairs are allowed to bind to their respective target proteins, if present in the sample. A PCR reporter sequence is formed by a proximity-dependent DNA polymerisation event. This is then amplified, and subsequently detected and quantified using real-time PCR. The method is validated and provides high sensitivity, specificity and scalability [5]. To investigate the utility of PEA technology for BALF samples obtained from patients with ILDs, we performed an explorative study to see if the proteome signature differed in the systemic and lung compartments between IPF and sarcoidosis, two ILDs with differing predominant mechanisms: fibrotic versus immunoinflammatory.

A total of 25 newly diagnosed ILD patients, including 15 patients with acute sarcoidosis (Löfgren syndrome) (median age 40 years (range 25-56 years), eight females) and 10 patients with IPF (median age 71 years (range 60-80 years), one female), were studied. Sarcoidosis and IPF were diagnosed in accordance with international recommendations $[2,3]$. Peripheral blood and BALF samples were collected from all study participants. Bronchoscopy with BALF was performed in accordance with the Polish Respiratory Society recommendations [6]. Both serum and BALF supernatant samples were frozen at $-80^{\circ} \mathrm{C}$ until further proteomic evaluation. Biological samples were analysed with a dedicated proteomics panel for 92 inflammatory proteins using PEA technology (Olink Proteomics, Uppsala, Sweden). For validation information of the protein measurements included in the Olink Inflammation panel see [7]. The complete

@ERSpublications

Proximity extension assay proves feasible for multiplex analysis of proteins in bronchoalveolar lavage. Small exploratory study found multiple inflammatory proteins that differed between patients with sarcoidosis and idiopathic pulmonary fibrosis. https://bit.ly/3nW14nF

Cite this article as: Majewski S, Zhou X, Miłkowska-Dymanowska J, et al. Proteomic profiling of peripheral blood and bronchoalveolar lavage fluid in interstitial lung diseases: an explorative study. ERJ Open Res 2021; 7: 00489-2020 [https://doi.org/10.1183/23120541.00489-2020].

Copyright (CThe authors 2021. This version is distributed under the terms of the Creative Commons Attribution NonCommercial Licence 4.0. For commercial reproduction rights and permissions contact permissions@ersnet.org 
list of analysed proteins can be found on the Olink Proteomics web page for the Inflammation panel (www.olink.com/products/inflammation/biomarkers/). The study protocol was approved by the Ethics Committee of the Medical University of Lodz and the study was conducted in accordance with the Declaration of Helsinki. All participants gave written informed consent for study participation.

For serum, a total of 81 out of 92 proteins were detectable (levels above the limit of detection in at least $75 \%$ of individuals). For BALF, the corresponding number was 49 out of 92 proteins (figures 1 and 2). All samples passed quality control except one BALF sample from a patient with sarcoidosis. The protein measurements from this BALF sample were removed from further analyses.

Multiple linear regression models were used with each of the 130 proteins (log-transformed) as dependent variables, a patient group (sarcoidosis or IPF) as an independent variable, and adjusted for age, sex, smoking status, and the percentage of lymphocytes in BALF. The results were adjusted for multiple testing using the Benjamini-Hochberg method to control for false discovery rate (FDR) [8]. The significance was set to FDR-corrected p-values $<0.05$.

Significant differences (adjusted for sex, age, smoking status, and lymphocyte percentage) were found for the following 16 proteins in BALF: matrix metalloproteinase (MMP)-1, MMP-10, interleukin (IL)-6, IL-8, transforming growth factor-alpha (TGF- $\alpha$ ), tumour necrosis factor (TNF)-related apoptosis-inducing ligand (TRAIL), osteoprotegerin (OPG), oncostatin M (OSM), chemokine (C-X-C motif) ligand (CXCL) 5,
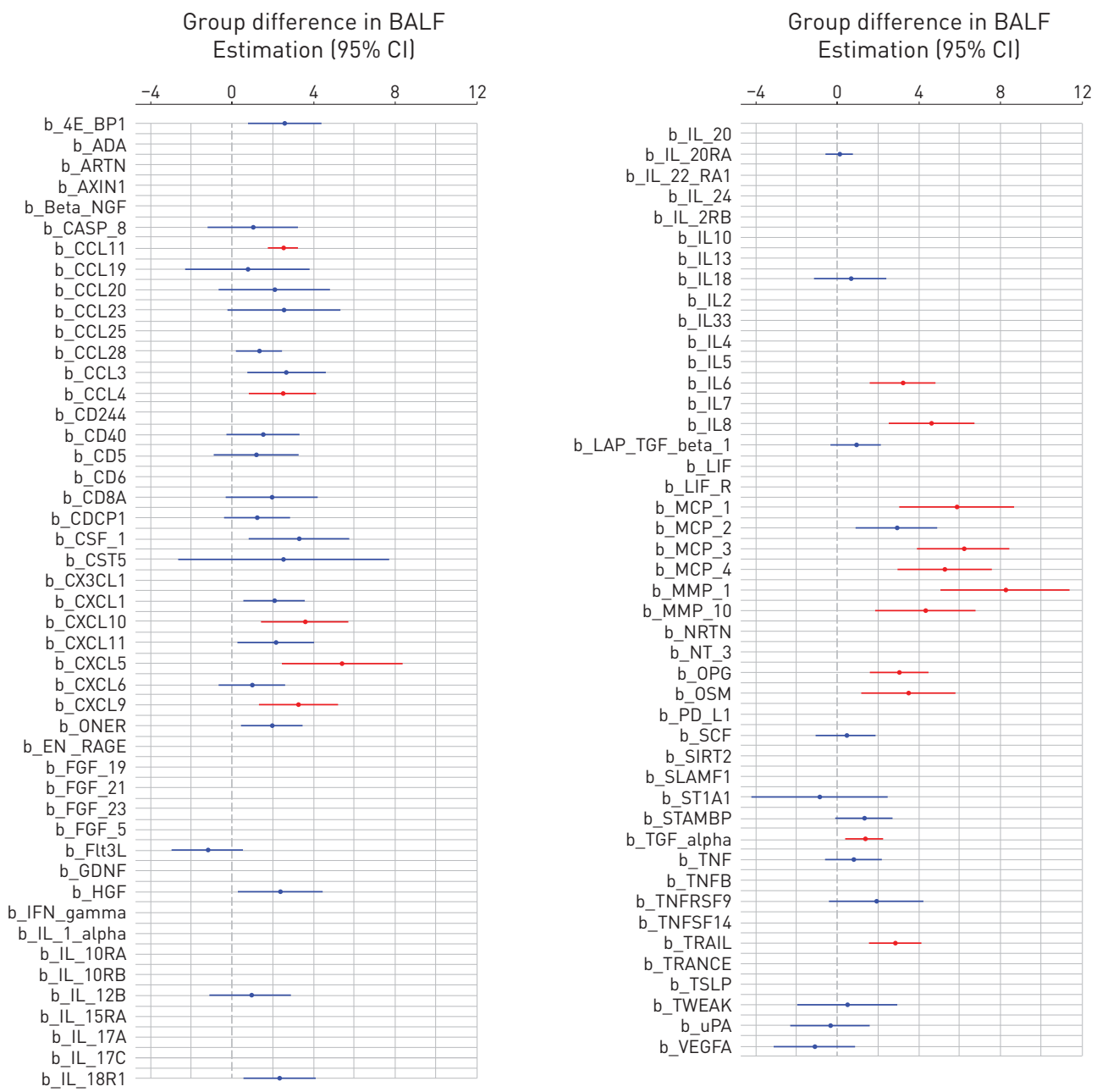

FIGURE 1 Differences in the expression levels of the 92 investigated proteins between the groups with idiopathic pulmonary fibrosis (IPF) and sarcoidosis expressed as regression coefficient (circle) and $95 \%$ confidence interval (line) in bronchoalveolar lavage fluid (BALF) (figure 2 displays the same data in serum). Significant differences (false discovery rate-corrected $p$-values $<0.05$ ) are plotted in red. If the confidence interval is on the right side of the dotted line (0) this means that subjects with IPF had higher levels of that protein than subjects with sarcoidosis. The list of the proteins can be found at https://www.olink.com/ products/inflammation/ 

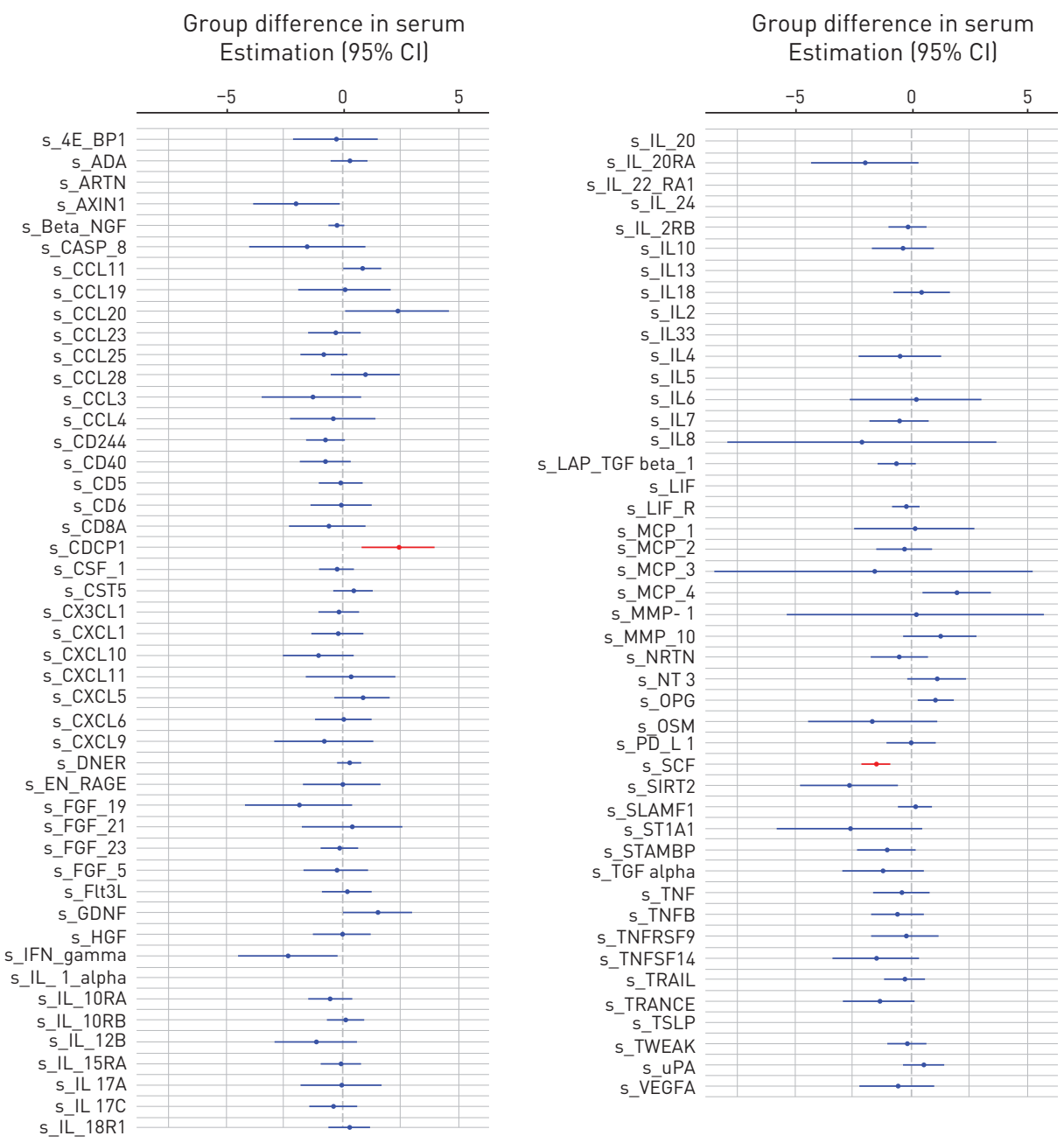

FIGURE 2 Differences in the expression levels of the 92 investigated proteins between the groups with idiopathic pulmonary fibrosis (IPF) and sarcoidosis expressed as regression coefficient (circle) and $95 \%$ confidence interval (line) in serum. Significant differences (false discovery rate-corrected $p$-values $<0.05$ ) are plotted in red. If the confidence interval is on the right side of the dotted line (0) this means that subjects with IPF had higher levels of that protein than subjects with sarcoidosis. The list of the proteins can be found at https://www.olink.com/products/inflammation/

CXCL9, CXCL10, monocyte chemoattractant protein (MCP)-1, MCP-3, MCP-4, macrophage inflammatory protein (MIP)-1 $\beta$, and eotaxin-1; and for two proteins in serum: stem cell factor (SCF) and CUB-domain containing protein 1 (CDCP1) (figure 1 and 2). All differentially expressed proteins had higher levels in IPF than sarcoidosis, except SCF (figure 1 and 2).

Pearson's correlation coefficients between the levels of proteins and the percentage of lymphocytes in the sarcoidosis group were tested. A total of 22 proteins were significantly related after adjustments for multiple testing: OSM, IL-12 $\beta$, IL-18, CXCL-11, T-cell surface glycoproteins CD5 and CD8 $\alpha$-chain, and TNF receptor superfamily member 9 (TNFRSF9) (all proteins measured in BALF, all $r$ values were between 0.8 and 1.0), CXCL-9, CXCL-10, MCP-2, MCP-4, C-C motif chemokine (CCL)3, CCL4, CCL19, IL-18, IL-18 receptor 1 (IL-18R1) and TNF- $\beta$ (IL-18 and TNF- $\beta$ from serum, others from BALF, $r$ values 0.7-0.8), CCL20, TRAIL, OPG, TNF, and hepatocyte growth factor (HGF) (all proteins measured in BALF, $r<0.7$ ).

Pearson's correlation coefficients between the levels of proteins in BALF and serum were tested for the proteins that could be detected in both BALF and serum $(n=49)$. One protein was significantly related after adjustments for multiple testing: CCL19.

In the present study, we successfully demonstrated the utility of PEA technology for comprehensive proteomics of BALF and identified differences in the proteome from the lung compartment between a systemic immunoinflammatory ILD, sarcoidosis, and a fibrotic ILD, IPF. Only two of the 81 measurable proteins in serum differed significantly between sarcoidosis and IPF: SCF and CDCP1. SCF may be crucial 
for the regulation of the inflammatory response in the lungs of patients with ILDs [9]. Recent studies suggest that cell surface glycoprotein CDCP1 acts as co-receptor of the TGF- $\beta 1$ signalling pathway [10]; however, its precise role in fibroproliferative diseases remains to be elucidated.

By contrast, almost one third (16 out of 49) of the measurable proteins in BALF differed significantly between sarcoidosis and IPF in our study. Our finding of increased MMP-1 in BALF of patients with IPF is in line with the previous findings of elevated MMP-1 levels in serum, BALF and lung tissue in IPF, compared with controls and other forms of ILDs [11]. Similarly, increased levels of IL-6 and IL-8 were previously found in patients with various ILDs compared with controls [12]. We noted overexpression of TRAIL, OPG and OSM in BALF from IPF subjects, which may suggest involvement in the TGF- $\beta 1$-independent accumulation of ECM proteins in IPF [13-15]. We also noted a number of upregulated CC and CXC chemokines, which may play significant roles in cell trafficking in pulmonary fibrosis [16].

Further research should include other fibroproliferative ILDs to check whether there are quantitative differences in the proteomes within that heterogeneous group. It seems that PEA technology offers a good alternative to mass spectrometry for proteomics studies utilising different biological samples, including BALF, and may be applied to study a larger population of patients with ILDs. Some of the proteins of interest might show a different profile if plasma is used instead of serum, but this was outside the scope of the present analyses. The small sample size of the two investigated groups might explain the large confidence intervals noted for the different proteins.

In summary, this small explorative study demonstrates, for the first time, the feasibility and usefulness of PEA technology for BALF proteomics in patients with ILDs. Despite the small sample size, we noted significant differences in levels of BALF proteins between subjects with IPF and those with sarcoidosis. Future, larger, prospective studies should focus on the biological relevance and clinical significance of our findings, with regard to disease prognosis, response to therapy, or novel pathophysiological pathways in ILDs.

Sebastian Majewski $\circledast^{1}$, Xingwu Zhou ${ }^{2,3}$, Joanna Miłkowska-Dymanowska $\oplus^{1}$, Adam J. Białas ${ }^{4}$, Wojciech J. Piotrowski ${ }^{1}$ and Andrei Malinovschi $\odot^{2}$

${ }^{1}$ Dept of Pneumology and Allergy, Medical University of Lodz, Lodz, Poland. ${ }^{2}$ Dept of Medical Sciences: Clinical Physiology, Uppsala University, Uppsala, Sweden. ${ }^{3}$ Dept of Medical Sciences: Respiratory Medicine, Sleep and Allergy, Uppsala University, Uppsala, Sweden. ${ }^{4}$ Dept of Pathobiology of Respiratory Diseases, Medical University of Lodz, Lodz, Poland.

Correspondence: Andrei Malinovschi, Dept of Medical Sciences: Clinical Physiology, Uppsala University, Akademiska sjukhuset Ing 35, 2tr. Uppsala 75185, Sweden. E-mail: andrei.malinovschi@medsci.uu.se

Received: 10 July 2020 | Accepted after revision: 3 Jan 2021

Support statement: This study was supported by: the Dept of Pneumology and Allergy, Medical University of Lodz, Poland; the Swedish Heart and Lung Foundation; and the Knut and Alice Wallenberg Foundation. Funding information for this article has been deposited with the Crossref Funder Registry.

Conflict of interest: None declared.

\section{References}

1 du Bois RM, Richeldi L, eds. Interstitial Lung Diseases (ERS Monograph). Sheffield, European Respiratory Society, 2009.

2 Raghu G, Remy-Jardin M, Myers JL, et al. Diagnosis of idiopathic pulmonary fibrosis. An official ATS/ERS/JRS/ ALAT clinical practice guideline. Am J Respir Crit Care Med 2018; 198: e44-e68.

3 Statement on sarcoidosis. Joint Statement of the American Thoracic Society (ATS), the European Respiratory Society (ERS) and the World Association of Sarcoidosis and Other Granulomatous Disorders (WASOG) adopted by the ATS Board of Directors and by the ERS Executive Committee, February 1999. Am J Respir Crit Care Med 1999; 160: 736-755.

4 Landi C, Bargagli E, Bianchi L, et al. Towards a functional proteomics approach to the comprehension of idiopathic pulmonary fibrosis, sarcoidosis, systemic sclerosis and pulmonary Langerhans cell histiocytosis. J Proteomics 2013; 83: 60-75.

5 Assarsson E, Lundberg M, Holmquist G, et al. Homogenous 96-plex PEA immunoassay exhibiting high sensitivity, specificity, and excellent scalability. PLoS One 2014; 9: e95192.

6 Chciałowski A, Chorostowska-Wynimko J, Fal A, et al. [Recommendation of the Polish Respiratory Society for bronchoalveolar lavage (BAL) sampling, processing and analysis methods]. Pneumonol Alergol Pol 2011; 79: 75-89.

7 Olink Proteomics. Olink Inflammation - Validation Data. Article number: 95302. https://www.olink.com/content/ uploads/2019/04/Olink-Inflammation-Validation-Data-v3.0.pdf Date last updated: 29 March 2019.

8 Haynes W. Benjamini-Hochberg Method. In: Dubitzky W, Wolkenhauer O, Cho K-H, et al., eds. Encyclopedia of Systems Biology [Internet]. New York, Springer New York, 2013; pp. 78-78. https://doi.org/10.1007/978-1-44199863-7_1215 
9 Fireman E, Kivity S, Shahar I, et al. Secretion of stem cell factor by alveolar fibroblasts in interstitial lung diseases. Immunol Lett 1999; 67: 229-236.

10 Predes D, Cruz JVR, Abreu JG, et al. CUB domain-containing protein 1 (CDCP1) binds transforming growth factor beta family members and increase TGF- $\beta 1$ signaling pathway. Exp Cell Res 2019; 383: 111499.

11 Rosas IO, Richards TJ, Konishi K, et al. MMP1 and MMP7 as potential peripheral blood biomarkers in idiopathic pulmonary fibrosis. PLoS Med 2008; 5: e93.

12 Takizawa H, Satoh M, Okazaki H, et al. Increased IL-6 and IL-8 in bronchoalveolar lavage fluids (BALF) from patients with sarcoidosis: correlation with the clinical parameters. Clin Exp Immunol 1997; 107: 175-181.

13 Collison AM, Li J, de Siqueira AP, et al. TRAIL signals through the ubiquitin ligase MID1 to promote pulmonary fibrosis. BMC Pulm Med 2019; 19: 31.

14 Habibie H, Burgess J, Wisman M, et al. Serum osteoprotegerin and fibulin-1 levels negatively correlate with DLCO in IPF. Eur Respir J 2019; 54: Suppl. 63, PA4702.

15 Wong S, Botelho FM, Rodrigues RM, et al. Oncostatin M overexpression induces matrix deposition, STAT3 activation, and SMAD1 dysregulation in lungs of fibrosis-resistant BALB/c mice. Lab Invest 2014; 94: 1003-1016.

16 Keane MP. The role of chemokines and cytokines in lung fibrosis. Eur Respir Rev 2008; 17: 151-156. 